\title{
Development of a 3D Multi-Parameter Method to Evaluate Heart Looping and Chamber Volume in Zebrafish Embryos
}

\author{
Allan P.S. Renom, Sarah E. Webb, Andrew L. Miller* \\ Division of Life Science and State Key Laboratory for Molecular Neuroscience, The Hong Kong University of \\ Science and Technology, Clear Water Bay, Kowloon, Hong Kong. \\ *Corresponding Author: Andrew L. Miller, Division of Life Science and State Key Laboratory for \\ Molecular Neuroscience, The Hong Kong University of Science and Technology, Clear Water Bay, \\ Kowloon, Hong Kong.
}

\begin{abstract}
Heart disease remains a leading cause of death worldwide. Some congenital cardiac malformations can result from defects in heart looping, which is a key process that ensures the correct alignment of the future chambers. The zebrafish is a widely used model for studying this essential developmental process; however, there is a lack of a standardized methodology to quantitatively characterize the heart looping process. Here, we present a standardized method for improving the accuracy when estimating the degree of heart looping using the zebrafish as a model. Our method relies on access to a lightsheet microscope, and $3 D$ analysis software, and it provides a means to acquire multi-parameter measurements related to shape changes during the heart looping process. This simple and more accurate approach can be used in future studies, which attempt to quantify heart-looping defects as well as aid in the elucidation of components of the signal transduction pathways that regulate this process.
\end{abstract}

Keywords: heart looping, light-sheet microscopy, 3D reconstruction, zebrafish

\section{INTRODUCTION}

Despite recent medical advancements, heart disease remains a leading cause of death worldwide. In the United States alone, one in every four deaths is due to heart disease [1]. This statistic is similar to the world population, where it was estimated in 2016 that $31 \%$ of the population died due to heart disease [2]. Cardiac development is a complex mechanism composed of a number of different morphogenetic processes. One of these processes, called 'heart looping' is crucial for ensuring that the future heart chambers are aligned correctly [3]. Indeed, in cases when heart looping is defective, then this leads to congenital cardiac malformations [4]. Therefore, studying the heart looping process should help reveal the diverse causes that contribute to the development of congenital heart disease and facilitate the development of potential treatments.

The zebrafish is a widely-used model for studying cardiovascular developmental processes due to its rapid ex-utero development, its genetic amenability, and the transparency of the early embryos and larvae, as well as the fact that it has a relatively simple two-chambered heart [5]. In zebrafish, the heart looping process begins at $\sim 22 \mathrm{hpf}$ with the formation of a linear heart tube (LHT) [5-7]. Shortly after it forms, the LHT is displaced leftward relative to the dorsal midline of the embryo, and then starting at $\sim 26 \mathrm{hpf}$, it undergoes bending and twisting movements in a process called heart looping [59]. During the latter stages of heart looping, the volume of the nascent cardiac chambers increase in a process known as 'ballooning'. This results in the formation of a two-chambered heart with distinct asymmetries between the atrial and the ventricular chambers [5,10]. A consequence of this morphogenetic process is the formation of the atrioventricular canal (AVC) connecting the atrium to the ventricle and the development of the distinctive heart shape. The expansion of both chambers is accomplished by the recruitment of myocardial cells from the second heart field. These cells will 
Development of a 3D Multi-Parameter Method to Evaluate Heart Looping and Chamber Volume in Zebrafish Embryos

contribute to the development of the sinus node at the venous pole and the outflow tract at the arterial pole $[5,7,11]$.

Although heart looping is a key process in cardiac development, many of the molecular, mechanical, and physiological processes that initiate, regulate, drive and terminate this process remain unclear [1216]. In addition, there is currently a lack of a standardized methodology to accurately estimate the extent of heart looping. This is the case for heart looping in normally developing control embryos, never mind in embryos where the looping process is challenged by genetic, molecular, or pharmacological manipulation in order to decipher regulatory processes. Indeed, many past and current studies rely only on a qualitative assessment with no quantitative evaluation [17-21]. Only a few recent publications have characterized the extent of heart looping via quantitative parameters such as the distance between the sinus venosus (SV) and the bulbus arteriosus (BA) [22-24], or by comparing the distance between the atrial and ventricular apex, with the entire length of the heart [25]. The latter generates an index value, which is inversely proportional to the extent of atrium/ventricle overlap such that as looping progresses then this value decreases [25]. The most commonly used quantitative parameter is the looping angle, represented by the angle between the anterior-posterior fish axis plane and the AVC plane [26-30]. However, some looping angles have also been calculated via less accurate methods such as measuring the angle between the atrium and the ventricle [31], or the angle between the atrial and ventricular axis [32]. Furthermore, most of the current volume measuring methods are restricted to 2D, which give only an approximation of the volume [33]. In summary, the current methods applied to quantitatively assess the extent of heart looping have been mostly limited to the measurement of a single parameter in 2D. However, we suggest that to more accurately estimate the extent of heart looping, multidimensional measurements are required in 3D. Here, we present a method to more accurately quantify heart looping using 3D light-sheet microscopy, and the measurement of four heart shape parameters: (1) Chamber volume; (2) SV-BA distance; (3) Chamber overlap index; and (4) Looping angle.

\section{Materials AND Methods}

\subsection{Zebrafish Husbandry and Embryo Collection}

The $\operatorname{Tg}((\mathrm{gal} 4) \mathrm{cmlc2}$ :GFP) transgenic line of zebrafish were maintained and their fertilized eggs were collected as previously described [34]. These fish, which express GFP in the heart via the cardiac myosin light chain 2 ( $\mathrm{cmlc}$ ) promoter, were obtained from the Zebrafish International Resource Centre (University of Oregon, Eugene, OR, USA). Fertilized eggs were maintained in Danieau's solution $\left(19.3 \mathrm{mM} \mathrm{NaCl}, 0.23 \mathrm{mM} \mathrm{KCl}, 0.13 \mathrm{mM} \mathrm{MgSO} \cdot \mathrm{MH}_{2} \mathrm{O}, 0.2 \mathrm{mM} \mathrm{Ca}\left(\mathrm{NO}_{3}\right)_{2}\right.$ and $1.67 \mathrm{mM}$ Hepes; pH 7.2) in a modified incubator (My Temp Mini Digital Incubator H2200-HC, Benchmark Scientific, NJ, USA) fitted with $14 \mathrm{~h}$ light/ $10 \mathrm{~h}$ dark cycle illumination at $\sim 28^{\circ} \mathrm{C}$ throughout most experiments until the desired developmental stage was reached [35,36]. The embryos were then anesthetized and the heart contractions were stopped by incubation in Danieau's solution containing $\sim 0.02 \%$ MS-222 (Sigma-Aldrich Corp. St. Louis, MO, USA) and $10 \mu \mathrm{M}$ 2,3-butanedione 2monoxime (BDM, Sigma-Aldrich Corp.) for $2 \mathrm{~min}$, after which the embryos were fixed with phosphate-buffered saline (PBS; $137 \mathrm{mM} \mathrm{NaCl}, 2.7 \mathrm{mM} \mathrm{KCl}, 16 \mathrm{mM} \mathrm{Na} \mathrm{HPO}_{4}, 4 \mathrm{mM}$ $\mathrm{NaH}_{2} \mathrm{PO}_{4} .2 \mathrm{H}_{2} \mathrm{O}, \mathrm{pH}$ 7.3) containing $4 \%$ paraformaldehyde (Electron Microscopy Sciences, Hatfield, PA, USA) and $4 \%$ sucrose overnight at $4^{\circ} \mathrm{C}$. The paraformaldehyde solution was stored at $4{ }^{\circ} \mathrm{C}$ but was pre-warmed to room temperature before use to minimize structural artefacts during fixation. After fixation, embryos were washed thoroughly with PBS, after which they were stored at $4^{\circ} \mathrm{C}$ for up to 2 weeks. All the procedures used in this study with live fish were performed in accordance with the guidelines and regulations set out by the Animal Ethics Committee of the HKUST and by the Department of Health, Hong Kong. 
Development of a 3D Multi-Parameter Method to Evaluate Heart Looping and Chamber Volume in Zebrafish Embryos

\subsection{Bright-Field and Fluorescence Imaging}

To study heart morphogenesis during zebrafish development, bright-field and GFP fluorescence images were acquired of the anterior-most region of fish centering on the cardiac region. Images were collected with the fish in ventral and lateral orientations using a Ximea MQ013RG-E2 USB 3.0 camera mounted on a Nikon AZ100 Multizoom microscope using an AZ Plan Apo 4X/0.4 NA objective lens. GFP fluorescence was captured using $465 \mathrm{~nm}-500 \mathrm{~nm}$ excitation and $510 \mathrm{~nm}-560$ $\mathrm{nm}$ emission filters. Before imaging, embryos were dechorionated, anesthetized in Danieau's solution containing $\sim 0.02 \%$ MS-222 and $10 \mu \mathrm{M} \mathrm{BDM}$ for $1 \mathrm{~min}$ before being mounted in an imaging chamber [34].

\subsection{Light-Sheet Microscopy}

Transgenic embryos were imaged with a Z1 light-sheet microscope (Carl Zeiss AG, Oberkochen, Germany), located at the Hong Kong Polytechnic University University Research Facility in Life Science unit. Fixed embryos were immersed in $2 \%$ low gelling temperature agarose (Sigma Aldrich) and mounted in a glass capillary with a Teflon tip plunger (size 3, 1.5 mm, Green/701998 Carl Zeiss AG, Oberkochen, Germany). The capillary was installed in the microscope capillary holding system and placed in a sample chamber filled with PBS. The capillary was rotated so that the heart of the fish could be visualized in a ventral orientation. Images were acquired using a 20x W-Plan-Apochromat 20x/1.0 UV-VIS-4909000160 objective, and 2 sCMOS cameras (Pc0 Edge). The GFP signal was captured using $488 \mathrm{~nm}$ laser excitation. Images were acquired using a dual side acquisition mode and analyzed with the 3/4D image visualization and analysis software, IMARIS 9.3 (Bitplane AG, Zurich, Switzerland).

\subsubsection{Rendering of the Zebrafish Heart}

A 3D rendering of each heart was obtained using IMARIS. The shape of the heart was drawn by hand using the 'surface creation' tool and with the contour option, which allowed us to draw an outline at every $10^{\text {th }}$ optical section through the entire heart $\mathrm{z}$-stack. The automated $3 \mathrm{D}$ rendering tool of IMARIS displayed some limitations with respect to our application; for example, it is dependent on the GFP signal, which, due to the nature of the $\mathrm{cmlc} 2$ promotor, resulted in an incomplete heart shape. Each hand-drawn section was separated by $4.7 \mu \mathrm{m}$ as each Z-section was equal to $0.47 \mu \mathrm{m}$. An average of 45 sections were drawn and combined to create a 3D model of the heart.

\subsection{Image Analysis}

Following the generation of the 3D reconstructed images, the volume of the whole heart and of the individual chambers were obtained using IMARIS. In addition, the looping process was quantified via the analysis of three different parameters. (1) The looping angle was determined by calculating the angle between the plane of the anterior/posterior fish axis and the plane created by the position of the AVC. An angle close to $90^{\circ}$ indicates that the two heart chambers have not looped at all and remain in a near-linear arrangement along the anterior/posterior axis of the fish. During normal looping, this angle decreases as the two chambers become positioned next to each other. (2) The SV-BA distance is the distance between the sinus venosus (SV; where blood enters the atrium), and the bulbus arteriosus (BA; where blood exits the ventricle). Each region was defined manually, and the distance was calculated from the 3D images generated. (3) The final parameter measured as an indication of cardiac looping was to compare the distance between the atrial and ventricular apex (distance $y$ in Fig. 1Cei,1Ceii), with the entire length of the heart (distance $x$ in Fig. 1Cei,1Ceii). The index value $(x / y)$ is inversely proportional to the extent of chamber overlap that results from the heart looping process [25]. A low index value represents a well-looped heart where the chambers show a high degree of overlap, whereas a high index value indicates that the two chambers exhibit a more linear arrangement. 
Development of a 3D Multi-Parameter Method to Evaluate Heart Looping and Chamber Volume in Zebrafish Embryos
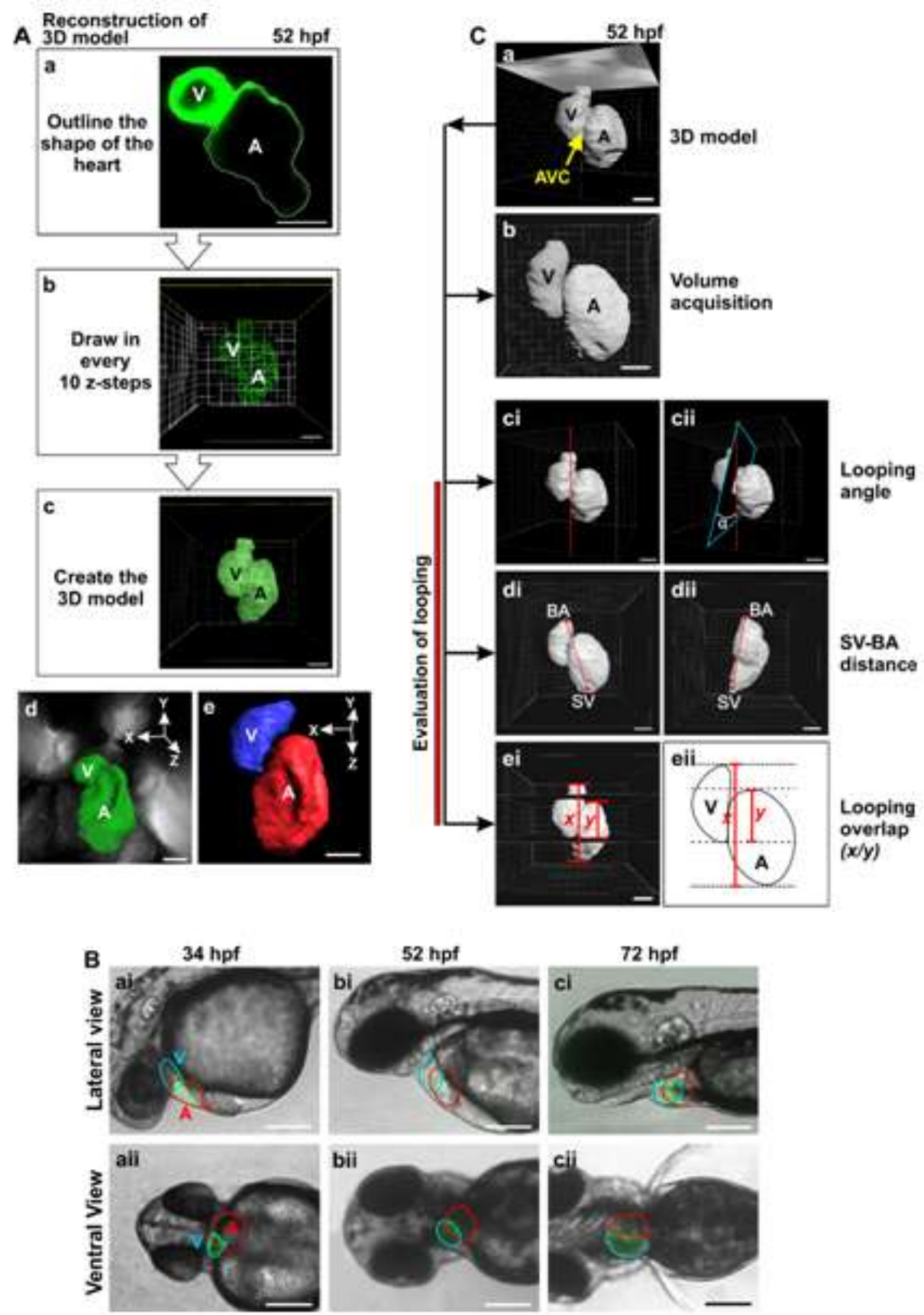

Fig1. Reconstruction of a 3D model of the heart of a zebrafish at $\sim 52$ hpf and the methods used to quantify various parameters at this stage of development. (A) Preparing the $3 D$ model. Using the Tg(cmlc2:GFP) fish line in conjunction with light-sheet microscopy, a series of z-sections was acquired through the heart. (Aa) This shows how the shape of the heart in one optical section was outlined using IMARIS. (Ab) The shape of the heart in every $10^{\text {th }}$ optical section of the z-stack was acquired to construct the overall $3 D$ heart shape. (Ac) The finished $3 D$ reconstruction of the heart. $(\boldsymbol{A d}, \boldsymbol{A e})$ Representative $3 D$ reconstructions showing (Ad) the heart viewed in a ventral orientation, and (Ae) the two heart chambers, such that the ventricle and atrium are represented in blue and red, respectively. (B) The position of the entire heart changes from $\sim 34$ hpf to $\sim 72$ hpf. These are bright-field and fluorescence images of $\mathrm{Tg}(\mathrm{cmlc} 2: \mathrm{GFP})$ embryos at (Ba) $\sim 34 \mathrm{hpf},(\boldsymbol{B b}) \sim 52 \mathrm{hpf}$ and (Bc) 72 hpf, taken from (Bai-Bci) lateral and (Baii-Bcii) ventral views. The regions bounded by the blue and red lines indicate the ventricle and atrium, respectively. $(\boldsymbol{C})$ Various parameters were measured using the rendered 3D model as shown in $(\boldsymbol{C a})$. (Cb) The volume of the heart as well as that of the ventricle and atrium alone were determined. (Cc-Ce) In addition, heart looping was characterized by calculating the (Cc) looping angle, $(\boldsymbol{C d})$ sinus venosus $(S V)$ to bulbus arteriosus $(B A)$ distance, and $(\boldsymbol{C e})$ level of looping overlap. With regards to the looping angle ( $\alpha$ ) (Ccii), this is defined as the angle between the fish body axis (shown by the red dashed line) and the atrioventricular canal plane (in blue). The looping overlap (Cei,Ceii) is the ratio of the total length of the heart $(x)$ with respect to the distance between the atrium and ventricle apex (y). $V, A$ and $A V C$ are ventricle, atrium, and atrioventricular canal, respectively. Scale bars are: $50 \mu m(\boldsymbol{A}, \boldsymbol{B})$ and $200 \mu m(\boldsymbol{C})$. 
Development of a 3D Multi-Parameter Method to Evaluate Heart Looping and Chamber Volume in Zebrafish Embryos

\subsection{Statistical Analysis}

All datasets were initially subjected to the Shapiro-Wilk test for the normality and the Levene's test for homogeneity of variance. If one of the above conditions was not fulfilled, then the dataset was analysed with the Mann Whitney U test. Data were considered to be significantly different when $P<0.05$. All statistical tests were performed with XLSTAT 2020 (Addinsoft). Figures and graphs were prepared using Corel DRAW Home \& Student 2018 version (Corel Corp., Ottawa, ON, Canada).

\section{RESULTS AND DISCUSSION}

The heart looping process is a key step in heart development. Thus, a defect in this process is often used to characterize heart developmental problems, such as those resulting from congenital defects. However, the quantitative evaluation of this complex, 3D developmental event is often missing, or it is estimated from a single measurable parameter. The first step we adopted in generating reliable quantitative data regarding heart looping was to generate 3D reconstructions of hearts from fixed fish using the IMARIS software (Fig. 1A). Following light-sheet microscopy to obtain z-stacks of optical sections, the shape of the heart was drawn by hand using the image of the heart visible via the fluorescence generated from GFP expression in the cardiac muscle cells (Fig. 1Aa). Such an outline was drawn every 10 sections of the z-stack taken through the heart (Fig. 1Ab). An average of 45 sections were drawn and combined to create each 3D reconstruction (Fig. 1Ac,1Ad). Following this method, each heart chamber could also be delineated based on the anatomy of the heart, and this allowed an estimation of their individual volumes (Fig. 1Ae).

An additional complication faced during the visualization of heart looping is that while looping progresses between $\sim 30 \mathrm{hpf}$ and $52 \mathrm{hpf}$, the zebrafish heart shifts from a left-sided body position at the heart tube stage $(\sim 30 \mathrm{hpf})$ to a cranial-ventral position at the same time that the major looping movements are underway (i.e., $>48 \mathrm{hpf}$ ) [25]. By $\sim 52 \mathrm{hpf}$, the two heart chambers are positioned (ventricle on the right and atrium on the left), approximately in a single plane perpendicular to the mid-sagittal body plane (Fig. 1B). Thus, during development, the overall position of the heart with respect to the embryonic anterior-posterior, dorsal-ventral, and left-right axes changes as a result of major morphogenetic rearrangements including head-lifting and axis-straightening. This is illustrated between $34 \mathrm{hpf}$ and $72 \mathrm{hpf}$ from a lateral (Fig. 1Bai-1Bci) and a ventral (Fig. 1Baii-1Bcii) view. The process of heart looping therefore occurs against a background of this organ rearrangement. This adds a considerable level of complexity when investigating the heart looping process from a quantitative perspective, and often leads to a large amount of variability when comparing published data. This is especially so, when these data are derived from 2D images, where only a single looping parameter is measured.

In this study, therefore, we applied a multi-dimensional approach based on 3D reconstruction from light-sheet fluorescent optical sections of the looping heart in zebrafish, by utilizing the $\operatorname{Tg}((\mathrm{gal}) \mathrm{cmlc}$ :GFP) line, which expresses GFP in the cardiac muscle driven by the $\mathrm{cmlc} 2$ promoter. One drawback to our method was that GFP expression, and thus fluorescent emission, was not homogenous throughout the entire heart tissue. However, we generated 3D reconstructions of zebrafish hearts by recording the heart shape every 10 optical sections along the z-axis for each heart examined. Each section was separated by $\sim 4.7 \mu \mathrm{m}$, and an average of 45 single optical sections were stacked together to create each 3D reconstruction. Following 3D reconstruction of whole hearts (Fig.1Ca) including their constituent chambers, various parameters could be quantified. These included the volume of the whole heart and individually of the atria and ventricles (Fig. 1Cb); the looping angle (Fig. 1Cci,1Ccii); the SV-BA distance (Fig. 1Cdi,1Cdii); and the looping overlap index (Fig. 1Cei,1Ceii). 
Development of a 3D Multi-Parameter Method to Evaluate Heart Looping and Chamber Volume in Zebrafish Embryos
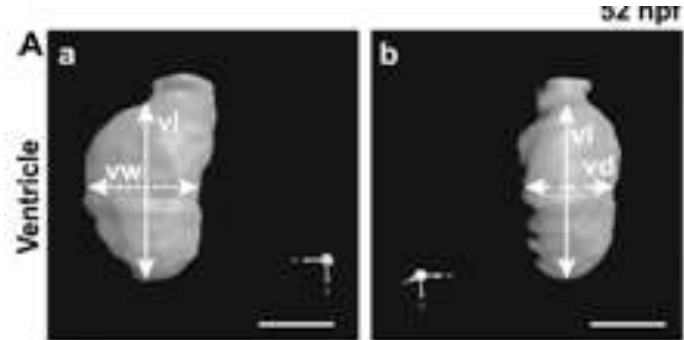

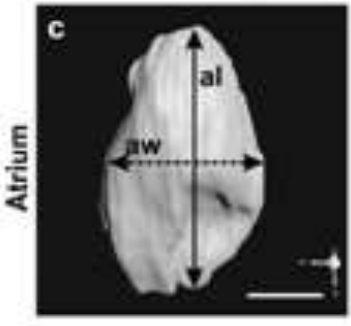

Ventral view

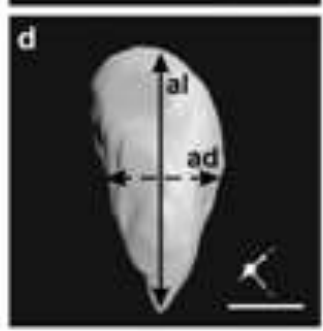

Lateral view
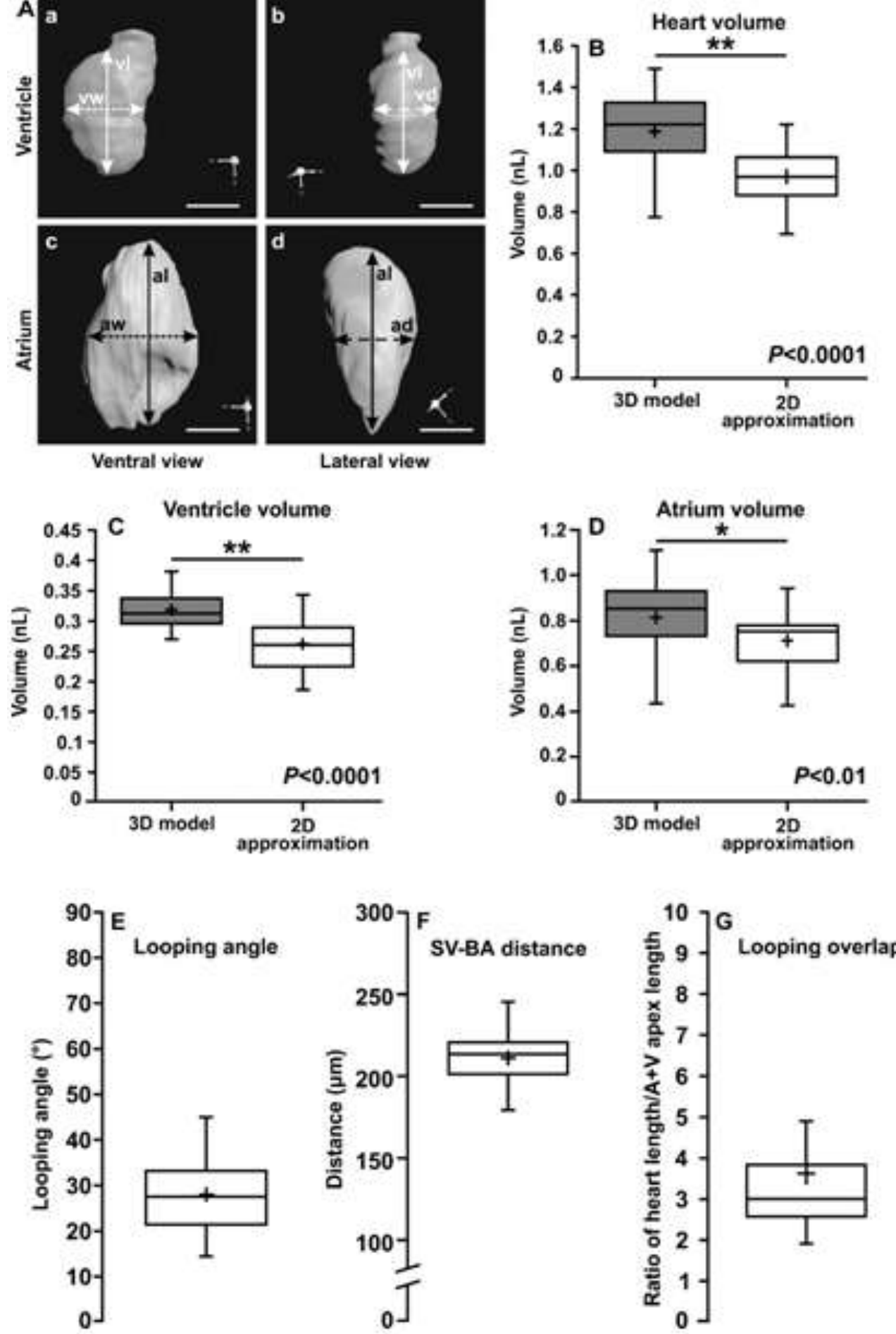

Fig2. Data analysis conducted on the heart of zebrafish larvae at $\sim 52$ hpf. (A-D) The volumes of the whole heart and of the ventricle and atrium alone were determined using our $3 D$ reconstruction method and with a $2 D$ approximation method, the latter which is based on the volume of an ellipse. (A) Representative $3 D$ models showing the $(\boldsymbol{A a}, \boldsymbol{A b})$ ventricle and $(\boldsymbol{A c}, \boldsymbol{A d})$ atrium of a 52 hpf zebrafish larva from (Aa,Ac) ventral and $(\boldsymbol{A b}, \boldsymbol{A d})$ lateral views. The labels indicate ventricle length $(v l)$, ventricle width $(v w)$, ventricle depth $(v d)$, atrial length (al), atrial width (aw), and atrial depth (ad). Scale bars are $50 \mu m .(B-D)$ Box plots comparing the volume of the $(\boldsymbol{B})$ whole heart and the $(\boldsymbol{C})$ ventricle and $(\boldsymbol{D})$ atrium calculated using the $3 D$ reconstruction and $2 D$ approximation methods. $(\boldsymbol{E}-\boldsymbol{G})$ Use of the $3 D$ reconstruction method to quantify the: $(\boldsymbol{E})$ Looping angle; $(\boldsymbol{F})$ sinus venosus to bulbus arteriosus $(S V-B A)$ distance; and $(\boldsymbol{G})$ looping overlap. In $(\boldsymbol{B}-\boldsymbol{G})$, each box indicates the range between the first quartile $\left(25^{\text {th }}\right.$ percentile $)$ and the third quartile $\left(75^{\text {th }}\right.$ percentile $)$, the line inside each box indicates the median value, and the crosses indicate the mean. The error bars indicate the maximum and minimum values, and in $(\boldsymbol{B}-\boldsymbol{D})$, the asterisks indicate $P<0.05$, determined by the Mann Whitney $U$ test. For each graph, $n=28$ fish larvae from 3 independent experiments.

International Journal of Research Studies in Biosciences (IJRSB)

Page | 39 
We analyzed and compared the volume of the whole heart along with the individual heart chambers by two different approaches in order to investigate whether they might generate similar results. Published zebrafish whole heart and chamber volumes are often estimated from $2 \mathrm{D}$ renditions, using ellipses as an approximation of the heart and chamber shape (i.e., ellipse volume formula: $4 / 3 \pi x$ (length/2) $\mathrm{x}($ width/2) $\mathrm{x}(\mathrm{depth} / 2))$ [36]. We compared the values obtained using this approach with the volumes calculated from our $3 \mathrm{D}$ reconstructions. The length, width, and depth of each chamber were obtained from the ventral and lateral views of the reconstructed heart (Fig. 2A). Ventricle volumes were found to be significantly different between the $2 \mathrm{D}$ and the $3 \mathrm{D}$ approaches. From the 3D reconstructions, the mean ventricle volume was $\sim 0.32 \mathrm{~nL}$ whereas with the $2 \mathrm{D}$ approximation, the mean was $\sim 0.26 \mathrm{~nL}$ (Fig. 2C). This difference represents an underestimation of $\sim 19 \%$. The atrium volumes were also significantly different between the $2 \mathrm{D}$ and the 3D approaches. With the 3D models, the mean atrium volume was $\sim 0.81 \mathrm{~nL}$, whereas, with the $2 \mathrm{D}$ approximation, the mean was $\sim 0.71 \mathrm{~nL}$ (Fig. 2D). This difference represents an underestimation of $\sim 13 \%$. The mean whole heart volume using the $2 \mathrm{D}$ approach was calculated by the addition of both chamber volumes. The heart volumes were significantly different between the 2D and the 3D approaches $(P<0.0001)$. With the 3D models, the mean heart volume was $\sim 1.19 \mathrm{~nL}$ whereas, with the $2 \mathrm{D}$ approximation, the mean was $\sim 0.97 \mathrm{~nL}$ (Fig. 2B). This difference represents an underestimation of $\sim 18 \%$. We suggest that the 3D approach represents a reasonable degree of fidelity and resolution with regards to a morphologically intact heart. We also propose that it provides a more accurate estimation of the dynamic changes in heart morphology that occur during the heart looping process - and in particular with respect to those that rely on 2D-based reconstructions of the heart. Moreover, in cases where the heart chamber shape is irregular, as in a number of mutant fish lines and heart disease states, we suggest that the 2D "ellipse approximation" method cannot be confidently applied, whereas our 3D method will improve the accuracy of these measurements.

We defined the looping angle $\alpha$, as the intersection of the fish body axis plane with the AVC plane (see the red dashed line and blue line, respectively in Fig. 1Ccii). This angle decreases as heart looping proceeds. At $52 \mathrm{hpf}$, we recorded a mean looping angle of $28^{\circ}$ (Fig. 2E). This result falls nicely in the range reported in the literature. For example, in a time window from $48 \mathrm{hpf}$ to $54 \mathrm{hpf}$, the heart looping angle has been reported to vary from $13^{\circ}$ to $30^{\circ}$ [26-30]. The SV-BA distance (Fig. 1Cdi and 1Cdii) is longer at the beginning of the looping process and decreases progressively as the initially linear heart structure loops around on itself. At $52 \mathrm{hpf}$ we recorded a mean SV-BA distance of $210 \mu \mathrm{m}$ (Fig. 2F). The looping overlap value at $52 \mathrm{hpf}$ was calculated to be $\sim 3.66 \pm 0.36$ (Fig. $2 \mathrm{G}$ ). Previous reports of the SV-BA distance in zebrafish vary considerably in the literature; for example, at $72 \mathrm{hpf}$ it ranges between $\sim 60 \mu \mathrm{m}$ and $\sim 150 \mu \mathrm{m}$ [23,37]. Our looping overlap value measured at 52 hpf was slightly greater than the value of $2.2 \pm 0.2$ previously reported for zebrafish heart looping at $54 \mathrm{hpf}$ [25]. However, as the overlap score is predicted to decrease as looping progresses [25], this suggests that from $\sim 52 \mathrm{hpf}$ to $\sim 54 \mathrm{hpf}$ heart looping might still being ongoing.

\section{CONClusion}

Here, we propose that the application of 3D heart reconstruction in combination with measuring multiple parameters associated with looping should result in a more robust and reliable estimation of the heart looping process.

\section{ACKNOWLEDGMENT}

We thank Dr Michael Yuen (University Research Facility in Life Science, Hong Kong Polytechnic University), for his technical support with the light-sheet microscope and Ms Mandy Chan (Division of Life Science, HKUST) for her advice and help during the experimental procedures. We also thank 
Profs King Lau Chow and Julie Semmelhack (Division of Life Science, HKUST) for their suggestions and advice regarding the project. This work was funded by a Hong Kong Research Grants Council General Research Fund award (\#16100115) and a Postgraduate Research/Teaching Studentship award from the Division of Life Science, HKUST.

\section{REFERENCES}

[1] Benjamin E.J., Muntne, P., Alonso A., Bittencour M.S., Callaway C.W., Carson A.P., Chamberlain A.M., Chang A.R., Cheng S., Das S.R., Delling F.N., Djousse L., Elkind M.S.V., Ferguson J.F., Fornage M., Chaffin Jordan L., Khan S.S., Kissela B.M., Knutson K.L., Kwan T.W., Lackland D.T., Lewis T.T., Lichtman J.H., Longenecker C.T. Loop M.S., Lutsey P.L., Martin S.S., Matsushita K., Moran A.E., Mussolina M.E., O’Flaherty M., Pandey A., Peral A.M., Rosamon W.D., Roth G.A., Sampson U.K.A., Satou G.M., Schroeder E.B., Shah S.H., Spartano N.L., Stokes A., Tirschwell D.L., Tsao C.W., Turakhia M.P., VanWagner L.B., Wilkins J.T., Wong S.S. and Virani S.S., Heart disease and stroke statistics-2019 update: A report from the American Heart Association. Circulation 139, e56-e528 (2019).

[2] World Health Organization (WHO). Cardiovascular diseases. 2017. https://www.who.int/news-room/factsheets/detail/cardiovascular-diseases-(cvds) (Accessed 26 April 2020).

[3] Noël E.S. and Bakkers J., Twists and turns. eLife 6, 32709 (2017)

[4] Männer J., The anatomy of cardiac looping: a step towards the understanding of the morphogenesis of several forms of congenital cardiac malformations. Clin. Anat. 22, 21-35(2009).

[5] Bakkers J., Zebrafish as a model to study cardiac development and human cardiac disease. Cardiovasc. Res. 91, 279-288 (2011).

[6] Dyer L.A. and Kirby M.L., The role of secondary heart field in cardiac development. Dev. Biol. 336, 137-144 (2009).

[7] Paige S.L., Plonowska K., Xu A. and Wu S.M., Molecular regulation of cardiomyocyte differentiation. Circ. Res. 116, 341-353 (2015).

[8] Smith K.A., Chocron S., von der Hardt S., de Pater E., Soufan A., Bussmann J., Schulte-Merker S., Hammerschmidt M. and Bakkers, J., Rotation and asymmetric development of the zebrafish heart requires directed migration of cardiac progenitor cells. Dev. Cell. 14, 287-297 (2008).

[9] Rohr S., Otten C. and Abdelilah-Seyfried S., Asymmetric involution of the myocardial field drives heart tube formation in zebrafish. Circ. Res. 102, E12-E19 (2008).

[10] Taber L.A., Biophysical mechanisms of cardiac looping. Int. J. Dev. Biol. 50, 323-332 (2006).

[11] Risebro C.A. and Riley P.R., Formation of the ventricles. Sci. World J. 6, 1862-1880 (2006).

[12] Soufan A.T., van den Berg G, Ruijter J.M., de Boer P.A.J., van den Hoff M.J.B. and Moorman A.F.M., Regionalized sequence of myocardial cell growth and proliferation characterizes early chamber formation. Circ. Res. 99, 545-552 (2006).

[13] Bussmann J., Bakkers J. and Schulte-Merker S., Early endocardial morphogenesis requires Sc1/Ta11. PLoS Genet. 3, e140 (2007).

[14] Holtzman N.G., Schoenebeck J.J., Tsai H.J. and Yelon D., Endocardium is necessary for cardiomyocyte movement during heart tube assembly. Dev. 134, 2379-2386 (2007).

[15] Dietrich A.C., Lombardo V.A., Veerkamp J., Priller F. and Abdelilah-Seyfried S., Blood flow and Bmp signaling control endocardial chamber morphogenesis. Dev. Cell 30, 367-377 (2014).

[16] Desgrange A., Le Garrec J.F. and Meilhac S.M., Left-right asymmetry in heart development and disease: forming the right loop. Dev. 145, 162776 (2018).

[17] Walton R.Z., Bruce A.E.E., Olivey H.E., Najib K., Johnson V., Earley J.U., Ho R.K. and Svensson E.C., Fogl is required for cardiac looping in zebrafish. Dev. Biol. 289, 482-493 (2006).

[18] Noël E.S., Verhoeven M., Lagendijk A.K., Tessadori F., Smith K., Choorapoikayil S., den Hertog J. and Bakkers, J.,A nodal-independent and tissue-intrinsic mechanism controls heart-looping chirality. Nat. Commun. 4, 2754 (2013).

[19] Li M., Hu X., Zhu J., Zhu C., Zhu S., Liu X., Han S. and Yu, Z., Overexpression of miR-19b impairs cardiac development in zebrafish by targeting ctnnb1. Cell Physiol. Biochem. 33, 1988-2002 (2014).

[20] Dash S.N., Narumanchi S., Paavola J., Perttunen S., Wang H., Lakkisto P., Tikkanen I. and Lehtonen S., Sept $7 b$ is required for the subcellular organization of cardiomyocytes and cardiac function in zebrafish. Am. J. Physiol. Heart Circ. Physiol. 312, 1085-1095 (2017). 
Development of a 3D Multi-Parameter Method to Evaluate Heart Looping and Chamber Volume in Zebrafish Embryos

[21] Song G., Han M., Li Z., Gan X., Chen X., Yang J., Dong S., Yan M., Wan J., Wang Y., Huang Z., Yin Z. and Zheng F., Deletion of Pr72 causes cardiac developmental defects in zebrafish. PLoS One 13, e0206883 (2018)

[22] Antkiewicz D.S., Burns C.G., Carney S.A., Peterson R.E. and Heideman W., Heart malformation is an early response to TCDD in embryonic zebrafish. Toxicol. Sci. 84, 368-377 (2005).

[23] Wu T.S., Yang J.J., Wang Y.W., Yu F.Y. and Liu B.H., Mycotoxin ochratoxin A disrupts renal development via a miR-731/prolactin receptor axis in zebrafish. Toxicol. Res. (Camb) 5, 519-529 (2016).

[24] Li J., Zhang Y., Liu K., He Q., Sun C., Han J., Han L. and Tian, Q., Xiaoaiping induces developmental toxicity in zebrafish embryos through activation of ER stress, apoptosis and the Wnt pathway. Front. Pharmacol. 9, 1250 (2018).

[25] Lombardo V.A., Heise M., Moghtadaei M., Bornhorst D., Männer J. and Abdelilah-Seyfried S., Morphogenetic control of zebrafish cardiac looping by Bmp signaling. Dev. 146, 180091 (2019).

[26] Chernyavskaya Y., Ebert A.M., Milligan E. and Garrity D.M.,Voltage-gated calcium channel CACNB2 $(\beta 2.1)$ protein is required in the heart for control of cell proliferation and heart tube integrity. Dev. Dyn. 241, 648-662 (2012).

[27] Parrie L.E., Renfrew E.M., Wal A.V., Mueller R.L. and Garrity D.M., Zebrafish tbx5 paralogs demonstrate independent essential requirements in cardiac and pectoral fin development. Dev. Dyn. 242, 485-502 (2013).

[28] Van Laarhoven P.M., Neitzel L.R., Quintana A.M., Geiger E.A., Zackai E.H., Clouthier D.E., Artinger K.B., Ming J.E. and Shaikh T.H., Kabuki syndrome genes KMT2D and KDM6A: functional analyses demonstrate critical roles in craniofacial, heart and brain development. Hum. Mol. Genet. 24, 4443-4453 (2015).

[29] Powell R., Bubenshchikova E., Fukuyo Y., Hsu C., Lakiza O., Nomura H., Renfrew E., Garrity D. and Obara T., Wtip is required for proepicardial organ specification and cardiac left/right asymmetry in zebrafish. Mol. Med. Rep.14, 2665-2678 (2016).

[30] Merks A.M., Swinarski M., Meyer A.M., Müller N.V., Özcan I., Donat S., Burger A., Gilbert S., Mosimann C., Abdelilah-Seyfried S. and Panáková D., Planar cell polarity signalling coordinates heart tube remodelling through tissue-scale polarisation of actomyosin activity. Nat. Commun. 9, 2161 (2018).

[31] Berndt C., Poschmann G., Stühler K., Holmgren A. and Bräutigam L., Zebrafish heart development is regulated via glutaredoxin 2 dependent migration and survival of neural crest cells. Redox Biol. 2, 673-678 (2014).

[32] Choudhry P. and Trede N.S., DiGeorge syndrome gene tbxl functions through wht1lr to regulate heart looping and differentiation. PLoS One 8, e58145 (2013).

[33] Folkesson M., Sadowska N., Vikingsson S., Karlsson M., Carlhäll C.-J., Länne T., Wågsäter D. and Jensen L., Differences in cardiovascular toxicities associated with cigarette smoking and snuff use revealed using novel zebrafish models. Biol. Open 5, 970-978 (2016).

[34] Cheung C.Y., Webb S.E., Love D.R. and Miller A.L.,Visualization, characterization and modulation of calcium signaling during the development of slow muscle cells in intact zebrafish embryos. Int. J. Dev. Biol. 55, 153-174 (2011).

[35] M. Westerfield, The Zebrafish Book. A Guide for the Laboratory Use of Zebrafish (Danio rerio). 4th ed. University of Oregon Press, Eugene, USA, 2000.

[36] Kimmel C.B., Ballard W.W., Kimmel S.R., Ullmann B. and Schilling T.F., Stages of embryonic development of the zebrafish. Dev. Dyn. 203, 253-310 (1995).

[37] Hoage T., Ding Y. and Xu X.,Quantifying cardiac functions in embryonic and adult zebrafish. Methods Mol. Biol. 843, 11-20 (2012).

[38] Bangeppagari M., Park S.H., Kundapur R.R. and Lee S.J., Graphene oxide induces cardiovascular defects in developing zebrafish (Danio rerio) embryo model: in-vivo toxicity assessment. Sci. Total Environ. 673, 810-820 (2019).

Citation: Andrew L. Miller, et al. "Development of a 3D Multi-Parameter Method to Evaluate Heart Looping and Chamber Volume in Zebrafish Embryos", International Journal of Research Studies in Biosciences, 8(4), pp. 34-42. DOI: https:// doi.org/10.20431/2349-0365.0804005

Copyright: (C) 2020 Authors, This is an open-access article distributed under the terms of the Creative Commons Attribution License, which permits unrestricted use, distribution, and reproduction in any medium, provided the original author and source are credited. 